\section{Epidemiologia do câncer de boca em laboratório público do Estado de Mato Grosso, Brasil}

\author{
Oral cancer epidemiology in a public laboratory \\ in Mato Grosso State, Brazil
}

\author{
1 Escola de Saúde Pública \\ do Estado de Mato Grosso, \\ Cuiabá, Brasil. \\ 2 Hospital do Câncer de Mato \\ de Grosso, Cuiabá, Brasil. \\ 3 Faculdade de Odontologia \\ de Araçatuba, Universidade \\ Estadual Paulista, Araçatuba, \\ Brasil. \\ ${ }_{4}$ MT Laboratório, Cuiabá, \\ Brasil. \\ Correspondência \\ F. T. Borges \\ Escola de Saúde Pública do \\ Estado de Mato Grosso. \\ Rua Adauto Botelho 552, \\ Cuiabá, MT \\ 78085-200, Brasil. \\ fabianotonaco@yahoo.com.br
}

\begin{abstract}
This study analyzed oral cancer epidemiology based on histopathology reports from a public laboratory in Mato Grosso, Brazil, after a specific policy was implemented for treating oral and facial diseases in the State. This was a crosssectional epidemiological study, based on 1,324 histopathology reports issued from January 2005 to December 2006. The study identified 44 cases of oral cancer, or $3 \%$ of all oral lesions, and analyzed them in relation to the following variables: histological type, gender, age, and patient's place of residence (capital versus rest of State). The most common histological type was squamous cell carcinoma. Most of the diagnoses occurred in men in their 50s and 60s living in the interior of the State. During two years in operation, this public oral pathology service had recorded a considerable number of oral cancer cases.
\end{abstract}

Mouth Neoplasms; Oral Health; Laboratories
Fabiano Tonaco Borges 1,2 Cléa Adas Saliba Garbin 3 Artur Aburad de Carvalhosa 2,4 Paulo Henrique de Souza Castro 2 Lídia Regina da Costa Hidalgo ${ }^{3}$

\section{Introdução}

O câncer de boca é o quinto tipo de câncer em incidência em todo o mundo, sendo muito freqüente na Ásia (Índia, Cingapura e outros países da região) representando mais de $50 \%$ de todos os diagnósticos de câncer 1 . Na Índia o câncer de boca é o tipo de neoplasia maligna mais incidente nos homens e ocupa a terceira posição entre as mulheres 1 .

Estudos relacionam a associação entre o câncer de boca e a pobreza, onde os indicadores de mortalidade e morbidade são ruins nas áreas de baixo nível sócio-econômico 2 . Sturgis 1, Oliveira et al. 3 e Carvalho et al. ${ }^{4}$ destacam que as características culturais do povo, o nível sócio-econômico da sociedade e o grau de acesso ao tratamento e tecnologia nos serviços públicos de saúde determinam a variação da incidência do câncer de boca no mundo. Em países desenvolvidos, o câncer de boca apresenta taxas de incidência e mortalidade menores quando comparados aos países em desenvolvimento ${ }^{4}$.

No Brasil, o câncer de boca representa o quinto tipo de câncer em incidência entre os homens e o sétimo entre as mulheres 5 . O Instituto Nacional de Câncer (INCA) estima para 2008 uma taxa bruta em homens e mulheres, respectivamente, de 11,00 e 3,88 casos novos por 100 mil habitantes (INCA http://www.inca. gov.br/estimativa $/ 2008 /$ index.asp?link=mapa asp\&ID=9, acessado em 10/Fev/2008; http:// 
www.inca.gov.br/estimativa/2008/index. asp?link=tabelaestados. asp\&UF=BR, acessado em 10/Fev/2008). Os maiores valores das taxas médias anuais de incidência, ajustadas por idade por 100 mil habitantes, foram constatadas no Rio de Janeiro (19,72 em homens e 6,26 em mulheres) (INCA. http://www.inca.gov.br/estimativa/2008/ index.asp?link=mapa. $\operatorname{asp} \& I D=9$, acessado em 10/Fev/2008). O INCA estimou para Mato Grosso, em 2008, 120 novos casos de câncer de boca, sendo 90 em homens e 30 em mulheres, consolidando uma taxa bruta de 6,25 em homens e 2,24 em mulheres (INCA. http://www.inca.gov.br/ estimativa $/ 2008 /$ index.asp?link=tabelaestados. asp\&UF=MT, acessado em 10/Fev/2008).

O Estado de Mato Grosso conta com 141 municípios distribuídos em $903.357 .908 \mathrm{~km}^{2}$ de extensão territorial, com uma população estimada de 2.803.274 em 2005 (Instituto Brasileiro de Geografia e Estatística - IBGE. http://www.ibge. gov.br/estadosat/perfil.php?sigla $=\mathrm{mt}, \quad$ acessado em 10/Fev/2008). Segundo dados do IBGE, o estado possui um total de 1.255 estabelecimentos públicos de saúde (IBGE. http://www.ibge.gov.br/ estadosat $/$ temas.php?sigla $=m t \&$ tema $=$ servicoss aude\&titulo=Servi\%E7os\%20de\%20sa\%FAde $\% 20$ 20005, acessado em 10/Fev/2008), que até o ano de 2004 não possuíam um serviço de patologia bucal no laboratório público (MT Laboratório), e nem mesmos um sistema de referência e contra referência para os casos suspeitos de câncer de boca.

A falta de um sistema de atenção ao câncer de boca no estado corroborou em tese, para o que se apresentassem no Hospital do Câncer de Mato Grosso (HCMT), pacientes com câncer de boca sem diagnóstico prévio e clinicamente em estádios avançados (estadiamento III e IV) 6. Este fato torna-se preocupante quando se analisam estudos como o de Leite \& Koifman 7, que ao estudarem a sobrevida dos pacientes com câncer de boca no INCA, no Rio de Janeiro, verificaram a correlação entre o estádio clínico e mortalidade, onde $18,1 \%$ dos pacientes no estádio I morreram, sendo que esta taxa sobe para $65,4 \%$ nos pacientes em estádio IV. Diante desses fatos, a detecção precoce do câncer de boca pela rede básica de saúde deve ser seriamente considerada ${ }^{8}$. A universalização do acesso aos meios de diagnóstico possibilitará a intervenção precoce ao câncer de boca.

Em 2004, o Estado de Mato Grosso publicou a política pública de atenção às doenças da boca e da face, por meio da Portaria $n^{o}$. 195, de 30 de novembro de 2004, da Secretaria de Estado de Saúde (SESMT) 9, instituindo um sistema de diagnóstico para as doenças da boca e da face, no âmbito da SESMT. Tais ações foram consolidadas pela Lei $n^{o} .8 .342$ de 30 de julho de 2005 10. A política pública de atenção às doenças da boca e da face foi publicada com as seguintes diretrizes: 1) Implantou o serviço de atenção às doenças da boca e da face no âmbito da SESMT, devendo atuar como referência estadual na realização de exames de diagnóstico de boca;

2) Instruiu os municípios na organização da rede de atenção básica para a realização de atividades preventivas e na realização de exames diagnósticos das lesões da boca e da face;

3) Determinou o MT Laboratório, da SESMT, como responsável no estado na realização de exames anátomo e histopatológico das lesões de boca e da face;

4) Criou um sistema de comunicação compulsório, pelos laboratórios de Mato Grosso, dos casos de câncer de boca ao programa de avaliação e vigilância do câncer da SESMT.

O objetivo deste trabalho foi analisar a prevalência dos casos de câncer de boca, diagnosticados no serviço público de patologia bucal, após a instituição da política de atenção às doenças da boca e da face do Estado de Mato Grosso, tornando públicos a experiência mato-grossense e os resultados preliminares da política pública.

\section{Metodologia}

Neste estudo realizou-se uma abordagem quantitativa por meio de uma pesquisa epidemiológica, documental, descritiva e transversal no banco de dados do laboratório público de Mato Grosso (MT Laboratório). O objeto estudado foi o laudo histopatológico, pesquisando-se um total de 1.324 laudos emitidos entre janeiro de 2005 e dezembro 2006. O laudo histopatológico foi considerado por Queiroz et al. 11 padrão ouro na confirmação do diagnóstico de câncer de boca. Analisaram-se a prevalência, o percentual do câncer de boca no universo das lesões bucais e as seguintes variáveis: tipo histológico, sexo, idade e procedência dos pacientes (capital ou do interior). Uma vez realizada a coleta e tabulação dos dados, realizou-se análise descritiva. O projeto foi submetido ao comitê de ética em pesquisa da Faculdade de Odontologia de Araçatuba da Universidade Estadual Paulista (FOA/UNESP), aprovado pelos processos FOA 2006-01605 e FOA 2006-01686.

\section{Resultados e discussão}

O primeiro ano de funcionamento da política pública de atenção às doenças da boca foi 2005 , não havendo registros anteriores de casos de lesões de boca no MT Laboratório. No primeiro 
ano foram encaminhadas ao MT Laboratório 358 lesões de boca, sendo que em 2006 registrou-se 966 lesões de boca, evidenciando um aumento de $269 \%$ entre 2005 e 2006 nos registros de lesões bucais. Os dois anos de funcionamento do serviço de patologia totalizaram 1.324 laudos diagnósticos, dos quais 44 casos (3\%) correspondiam ao câncer de boca (Figura 1).

Figura 1

Distribuição percentual dos casos de câncer de boca em relação ao universo das lesões diagnósticos. MT Laboratório, Mato Grosso, Brasil, 2005 e 2006.

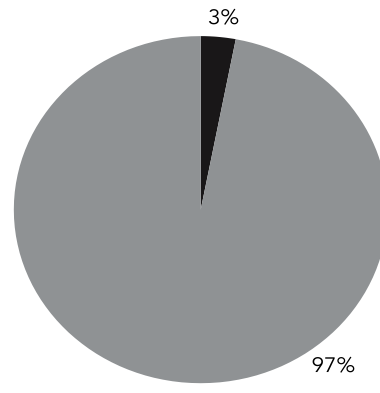

Câncer de boca

Outras lesões da boca
Moimaz et al. 12 verificaram a ocorrência de $1 \%$ de lesões malignas no universo das lesões de boca diagnosticadas em pacientes idosos no interior paulista. Corrêa et al. 13 analisaram 2.250 laudos histopatológicos de boca de pacientes, em dois grupos: pacientes idosos, com 60 anos ou mais, e não idosos, menos de 60 anos. Verificouse uma participação de $11,5 \%$ de lesões malignas epiteliais em pacientes idosos e 1,8\% de lesões malignas epiteliais em pacientes não idosos, no universo de todas as lesões estudadas.

Em 2006 registraram-se 32 casos de câncer de boca, num aumento de $266 \%$ nos registros de câncer entre 2005 e 2006 (Figura 2). Este aumento pode ser explicado pela subnotificação do câncer de boca em 2005, em decorrência da sedimentação do serviço. Riera \& Martínez 14 verificaram um aumento na taxa bruta dos casos de câncer de boca no Chile entre 1969 e 2002 de 3,0 para 5,3 por 100 mil habitantes. Wünsch-Filho 5 constatou um aumento na taxa bruta entre as mulheres entre 1983 e 1998 na cidade de São Paulo, Brasil.

A média de idade dos pacientes com câncer foi de 55,2 anos, entre a $5 \underline{a}$ e $6 \underline{a}$ décadas de vida (Figura 3), média próxima da verificada por Durazzo et al. 15, que analisaram 374 prontuários entre 1994 a 2002, constatando uma média de idade de 57,4 anos. Os pacientes com menos de 60 anos representam $68,1 \%$ e os com 60 anos ou mais, $31,9 \%$. Llewellyn et al. ${ }^{16}$ analisaram a ocorrência

Figura 2

Distribuição numérica dos casos de câncer de boca $(n=44)$ por gênero e por ano. MT Laboratório, Mato Grosso, Brasil, 2005 e 2006.

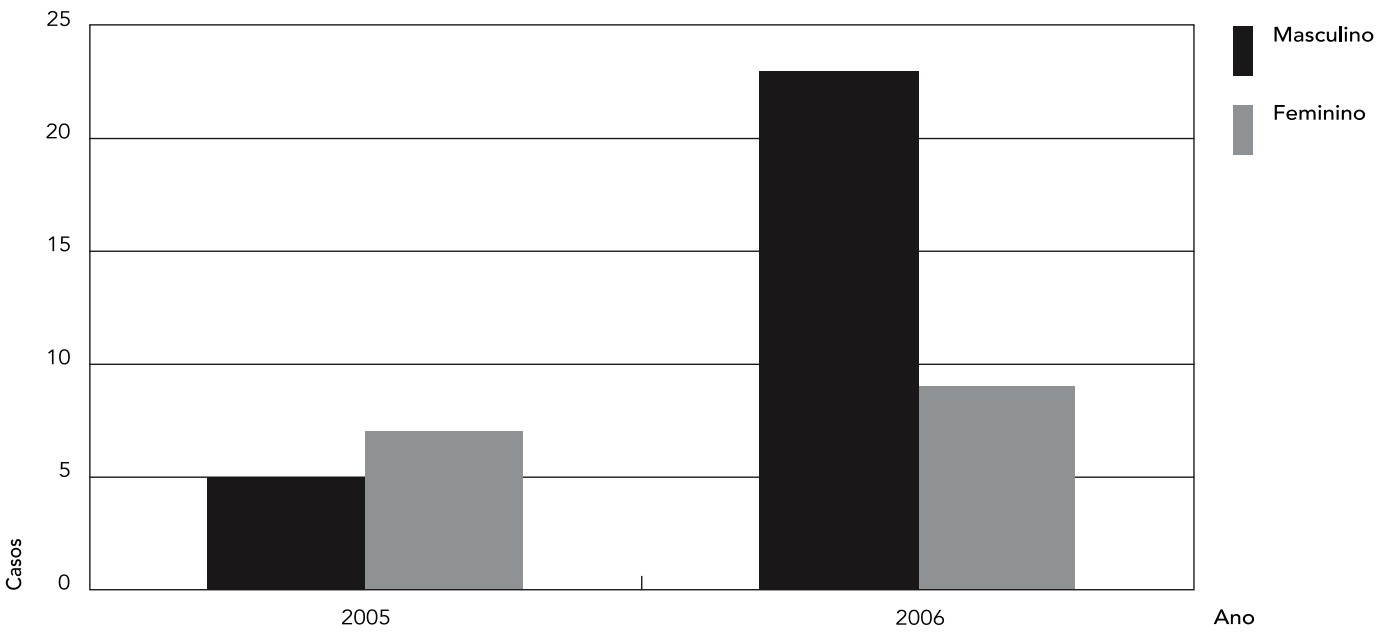


Figura 3

Distribuição percentual dos casos de câncer $(n=44)$ por década de vida mais incidente. MT Laboratório,

Mato Grosso, Brasil, 2005 e 2006.

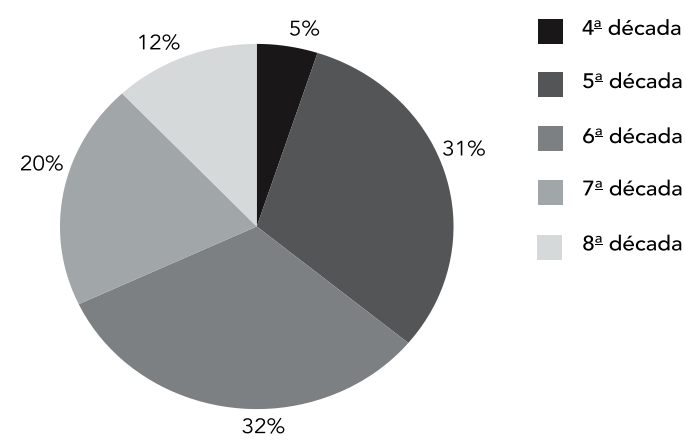

de câncer de boca em 116 pacientes no Reino Unido em pacientes com menos de 45 anos, e verificaram que $52 \%$ dos pacientes tinham entre 41 e 45 anos, ou seja, na $5^{\text {a }}$ década de vida. Os autores notaram na pesquisa a relação da ocorrência do câncer de boca em adultos jovens com exposição maior ao tabaco e ao álcool do que no grupo controle 16 .

O sexo feminino predominou em 2005 com $58,3 \%$ dos diagnósticos de câncer de boca. No ano de 2006 o sexo que predominou foi o masculino com $71,8 \%$. Este dado está próximo ao verificado na cidade do Rio de Janeiro por Leite \& Koifman 7 entre 1996 e 1997. Vários estudos que indicam o sexo masculino o mais acometido pelo câncer de boca 1,5,15,17 . Shafer et al. 18 em

Tabela 1

Distribuição numérica dos tipos histológicos de câncer de boca $(n=44)$ diagnosticados pelo MT Laboratório. Mato Grosso, Brasil, 2005 e 2006.

\begin{tabular}{lccc}
\hline Tipo histológico & $\mathbf{2 0 0 5}$ & $\mathbf{2 0 0 6}$ & Total \\
\hline Carcinoma epidermóide & 7 & 25 & 32 \\
Carcinoma basocelular & 2 & 4 & 6 \\
Carcinoma adenóide cístico & 1 & 1 & 2 \\
Carcinoma indiferenciado & 1 & 0 & 1 \\
Carcinoma muco-epidermóide & 1 & 0 & 1 \\
Adenocarcinoma & 0 & 1 & 1 \\
Ameloblastoma maligno & 0 & 1 & 1 \\
Total & 12 & 32 & 44 \\
\hline
\end{tabular}

1987 discorriam sobre a tendência à diminuição da diferença registrada na ocorrência do câncer de boca entre os dois sexos, principalmente devido ao fato de que certos hábitos masculinos provavelmente relacionados à etiologia da doença tenham sido adotados pelas mulheres, o que pode justificar, pelo menos em parte, a maior prevalência no gênero feminino. No estudo de Iamarron et al. 19 houve uma discreta predominância do câncer de boca nos homens com 57,3\% em relação às mulheres com 42,7\%. O mesmo fenômeno foi verificado por Hamada et al. 20 na cidade de Bombay, Índia, onde as taxas entre 1973 e 1975 foram idênticas.

O tipo histológico mais prevalente foi o carcinoma epidermóide nos dois anos estudados (Tabela 1) com 72,7\% dos casos de câncer de boca, em consonância com os estudos de Gervásio et al. ${ }^{21}$ e Corrêa et al. ${ }^{13}$. Oliveira et al. ${ }^{3}$ analisaram o perfil epidemiológico da cidade de São Paulo entre 1997 e 1999 e tiveram a mesma constatação, o mesmo ocorrendo no Chile, onde $90 \%$ dos casos de câncer de boca também corresponderam ao carcinoma epidermóide 14.

Ao analisar a procedência dos casos de câncer de boca verificou-se que a grande maioria procedia do interior do estado nos dois anos estudados (Figura 4).

Em uma análise retrospectiva de 740 casos de câncer de boca no Brasil Gervásio et al. 21 verificaram que a maior parte dos pacientes (36\%) tinha como ocupação o trabalho no campo, mesmo fato verificado por Güneri et al. 22 na Turquia. Mato Grosso é estado que tem sua economia com base na agricultura e na pecuária, com um grande contingente de trabalhadores rurais.

Figura 4

Procedência dos casos de câncer de boca $(n=44)$. Mato Grosso, Brasil, 2005 e 2006.

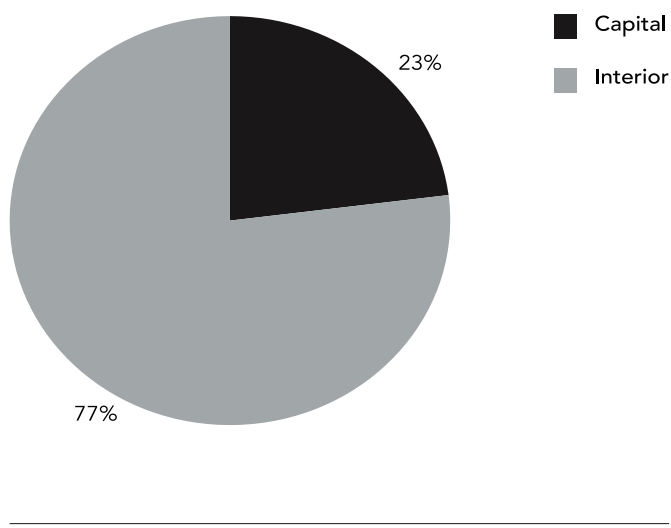




\section{Considerações finais}

Neste estudo os dados epidemiológicos mostraram a assimilação, por parte das unidades de saúde, do serviço de patologia bucal do MT Laboratório, que aumentou sua rotina em $269 \%$. O registro do câncer de boca no MT Laboratório au- mentou em $266 \%$ entre 2005 e 2006. Os homens em idade produtiva foram os mais acometidos pelo câncer de boca. A maioria dos pacientes procedia do interior do estado. Conclui-se com este estudo que em dois anos de funcionamento o serviço público de patologia registrou um considerável número de casos de câncer de boca.

\section{Resumo}

O objetivo deste trabalho foi analisar a epidemiologia do câncer de boca, dos casos diagnosticados pelo laboratório público do Estado de Mato Grosso, Brasil, após a instituição da política de atenção às doenças da boca e da face do estado. Trata-se de um estudo epidemiológico transversal. O objeto estudado foi o laudo histopatológico, pesquisando-se um total de 1.324 laudos emitidos entre janeiro de 2005 e dezembro 2006. Verificaram-se os casos de câncer de boca, o seu percentual em relação ao universo das lesões bucais e as seguintes variáveis: tipo histológico, sexo, idade e procedência dos pacientes (capital ou do interior). Após a análise dos dados verificaram-se 44 lesões de câncer de boca, representando $3 \%$ dos diagnósticos. O tipo histológico mais incidente foi o carcinoma epidermóide. A maioria dos diagnósticos foi referente aos homens na $5^{a}$ e $6^{a}$ décadas de vida residentes no interior do estado. Conclui-se com este estudo que em dois anos de funcionamento o serviço público de patologia bucal registrou um considerável número de casos de câncer de boca.

\section{Colaboradores}

Todos os autores participaram da construção do trabalho, do levantamento e análise dos dados e redação do texto.

\section{Agradecimentos}

L. R. C. Hidalgo é bolsista de iniciação científica do Conselho Nacional de Desenvolvimento Científico e Tecnológico (CNPq). 


\section{Referências}

1. Stugis EM. A review of social and behavioral efforts at oral cancer preventions in India. Head Neck 2004; 26:937-44.

2. Antunes JLF, Biazevic MGH, Araújo ME, Tomita NE, Chinellato, Narvai PC. Trends and spatial distribution of oral cancer mortality in São Paulo, Brazil, 1980-1998. Oral Oncol 2001; 37:345-50.

3. Oliveira EF, Silva OMP, Blachman IT, Pio MRB. Oral cancer epidemiological profile in São Paulo city. In: Varma AK, editor. Oral oncology. v. 10. New Delhi: Northern Book Centre; 2005. p. 13-21.

4. Carvalho LA, Singh B, Spiro RH, Kowalski LP, Shah JP. Cancer of the oral cavity: a comparison between institutions in a developing and a developed nation. Head Neck 2004; 26:31-8.

5. Wünsch-Filho V. The epidemiology of oral and pharynx cancer in Brazil. Oral Oncol 2002; 38: 737-46.

6. Garbin CAS, Moimaz SAS, Carvalhosa AA, Borges FT, Bezerra FV. Epidemiological survey of oral cancer cases in 2005 after the introduction of the carefulness at oral diseases politics in Mato Grosso State - Brazil In: Varma AK, Piemonte M, editors. Oral oncology. v. 11. New Delhi: Northern Book Centre; 2006. p. 70-3.

7. Leite ICG, Koifman S. Survival analysis in a sample of oral cancer patients at a reference hospital in Rio de Janeiro, Brazil. Oral Oncol 1998; 34:347-52.

8. Jitomirski F. Câncer bucal. In: Pinto VG, organizador. Saúde bucal coletiva. 4a Ed. São Paulo: Editora Santos; 2000. p. 445-56.

9. Mato Grosso. Portaria 195 de 30 de novembro de 2004: dispõe sobre a política estadual de atenção às doenças da boca e da face, no âmbito da Secretaria de Estado de Saúde de Mato Grosso - SES/ MT. Diário Oficial do Estado de Mato Grosso 2004; 30 nov.

10. Mato Grosso. Lei estadual 8342 de 30 de junho de 2005: institui a política estadual de atenção às doenças da boca e da face no âmbito da Secretaria de Estado de Saúde de Mato Grosso. Diário Oficial do Estado de Mato Grosso 2005; 30 jul.

11. Queiroz RCS, Mattos IE, Monteiro GTR, Koifman S. Confiabilidade e validade das declarações de óbito por câncer de boca no Município do Rio de Janeiro. Cad Saúde Pública 2003; 19:1645-53.

12. Moimaz SAS, Saliba NA, Antonio MNR. Prevention and diagnosis of the oral cancer in elderly people. In: Varma AK, editor. Oral oncology. v. 10. New Delhi: Northern Book Centre; 2005. p. 13-21.
13. Corrêa L, Frigerio MLMA, Souza SCOM, Novelli MD. Oral lesions in ederly population: a biopsy survey using 2250 histopatological records. Gerodontology 2006; 23:48-54.

14. Riera P, Martínez R. Morbilidad y mortalidad por cáncer oral y faríngeo en Chile. Rev Med Chile 2005; 133:555-63.

15. Durazzo MD, Araújo CE, Brandão Neto JS, Potenza AS, Costa P, Takeda F, et al. Clinical and epidemiological features of oral cancer in a medical school teaching hospital from 1994 to 2002: increasing incidence in women, predominance of advanced local disease, and low incidence of neck metastases. Clinics 2005; 60:293-300.

16. Llewellyn CD, Linklater K, Bell J, Johnson NW, Warnakulasuriya S. An analysis of risk factors for oral cancer in young people: a case-control study. Oral Oncol 2004; 40:304-13.

17. Luna-Ortiz K, Güemes-Meza A, VillavicencioValencia V, Mosqueda-Taylor A. Lip cancer experience in Mexico. An 11 year retrospective study. Oral Oncol 2004; 40:992-9.

18. Shafer WG, Hine MK, Levy BM. Tratado de patologia bucal. 4a Ed. Rio de Janeiro: Editora Guanabara Koogan; 1987.

19. Iamaroon A, Pattanaporn K, Pongsirwet S, Wanachantararak S, Prapayasatok S, Jittidecharaks S, et al. Analysis of 587 cases of oral squamous cell carcinoma in northern Thailand whit a focus on young people. Int J Oral Maxillofac Surg 2004; 33:84-8.

20. Hamada GS, Bös AJG, Kasuga H, Hirayama T. Comparative epidemiology of oral cancer in Brazil and India. Tokai J Exp Clin Med 1991; 16:63-72.

21. Gervasio OLAS, Dutra RA, Tartaglia SMA, Vasconcellos WA, Barbosa AA, Aguiar MC. Oral squamous cell carcinoma: a retrospective study of 740 cases in a Brazilian population. Braz Dent J 2001; 12: 57-61.

22. Güneri P, Cankaya H, Yanuzer A, Güneri EA, Erise L, Dzkul D, et al. Primary oral cancer in a Turkish population sample: association with sociodemographic features, smoking, alcohol, diet and dentition. Oral Oncol 2005; 41:1005-12.

Recebido em 30/Jul/2007

Versão final reapresentada em 18/Fev/2008 Aprovado em 28/Fev/2008 